\title{
Evolution of pattern of injury and quantitative MRI on days 1 and 3 in term newborns with hypoxic-ischemic encephalopathy
}

\author{
Dawn Gano', Vann Chau', Kenneth J. Poskitt' ${ }^{2}$ Alan Hill', Elke Roland' ${ }^{1}$, Rollin Brant ${ }^{3}$, Mark Chalmers' ${ }^{1}$ and Steven P. Miller ${ }^{1,4}$
}

BACKGROUND: Brain injury in term neonatal hypoxicischemic encephalopathy (HIE) emerges on magnetic resonance imaging (MRI) by day 3. This study aimed to address the relationship of $\mathrm{MRI}$, diffusion tensor imaging (DTI), and MR spectroscopic imaging (MRSI) findings on days 1 and 3 in a prospective cohort of term newborns with HIE.

METHODS: A total of 24 term newborns with HIE were prospectively studied with MRI on days 1 and 3; 19 were imaged with DTI and MRSI on days 1 and 3. MRI was assessed using validated scores. The relationship between MRI, DTI, and MRSI values on days 1 and 3 was determined using linear regression for repeated measures.

RESULTS: Conventional MRI showed a complex variation of findings from day 1 to 3 . In gray matter, mean diffusivity $\left(D_{\text {av }}\right)$ and metabolite ratios measured on day 1 were predictive of values on day 3 (all $P \leq 0.04$ ). In white matter, $D_{\text {av' }}$ fractional anisotropy (FA), and $\mathrm{N}$-acetylaspartate (NAA)/choline on days 1 and 3 were strongly related (all $P \leq 0.003$ ). Hypothermia appeared to attenuate the severity and progression of brain injury in the six treated newborns.

CONCLUSION: In term newborns with HIE, quantitative MR values on days 1 and 3 are strongly associated, providing an objective measure of injury before qualitative images.

$\mathbf{H}$ ypoxic-ischemic injury is the most common cause of neonatal encephalopathy. Neonatal encephalopathy occurs in 1-6 per 1,000 live births and results in significant morbidity and mortality $(1,2)$. The identification of accurate biomarkers of brain injury in newborns with encephalopathy has important implications for early diagnosis, potential initiation of neuroprotective therapy, and prognostication of neurodevelopment.

Brain injury in normothermic newborns with hypoxicischemic encephalopathy (HIE) is consistently evident on diffusion-weighted magnetic resonance imaging (DW-MRI) by the third day of life and evolves further over the first 2 wk of life (3). The extent of brain injury on MRI in newborns with HIE is an important predictor of neurodevelopmental outcome $(4,5)$. The predominant pattern of injury, which is established on conventional MRI by the second week of life, is more strongly associated with neurodevelopmental outcome than the severity of injury in any one region (5). As the process of injury unfolds in the first hours and days of life, findings on conventional MRI vary considerably (3). Consequently, prognostication based on the clinical MRI is limited in the initial days of life until the predominant pattern of injury is fully apparent.

Advanced MRI techniques, such as diffusion tensor imaging (DTI) and MR spectroscopic imaging (MRSI), have the potential to more accurately identify brain injury earlier in its progression $(6,7)$. Although several studies have measured quantitative parameters in newborns with HIE in the first few days of life $(3,4,8-13)$, it remains unknown whether quantitative parameters measured early are predictive of findings uniformly assessed at serial time points. The primary objective of the current study was to assess the relationship between DTI and MRSI findings on days 1 and 3 of life in a prospective cohort of term newborns with HIE. We also assessed the relationship between DTI and MRSI findings on days 1 and 10 , evaluated the progression of the predominant pattern of injury on conventional MRI, and explored the effects of systemic hypothermia on the evolution of brain injury in newborns with HIE.

\section{RESULTS}

\section{Clinical Features}

A total of 24 term newborns with HIE were studied prospectively with qualitative MRI on days 1 and 3 of life; 19 were also imaged with DTI and MRSI on days 1 and 3. Among the cohort, six newborns were treated with systemic hypothermia as part of a concomitant research protocol (14). There were no significant differences in the clinical characteristics between normothermic and hypothermic newborns, as summarized in Table 1.

\section{Pattern of Injury}

Conventional MRI demonstrated a complex variation of findings and change in the predominant pattern of injury between 
days 1, 3, and 10, as illustrated in Figures 1 and 2. Given the apparent variation in the progression of pattern of injury between normothermic and hypothermic newborns, these two groups will be described separately.

\section{Progression of Pattern of Injury in Normothermic Newborns}

The distribution of pattern of injury in normothermic newborns $(n=18)$ on day 3 of life included six normal, three basal nuclei (BN) predominant, one watershed (WS) predominant, five focal-multifocal injury, and three total injury. Six newborns had a major change in the pattern of injury between days 1 and 3. Two newborns with normal MRIs on day 1 developed a $\mathrm{BN}$ pattern on day 3 ; two newborns with a $\mathrm{BN}$ pattern on day 1 evolved to a pattern of total injury on day 3 . In addition, two newborns with normal MRIs on day 1 developed focal-multifocal injury on day 3 . The pattern of injury on day 10 was unchanged from day 3 in eight of nine normothermic newborns imaged on day 10, and one newborn had minor focal injury apparent after normal scanning performed on days 1 and 3.

\section{Progression of Pattern of Injury in Hypothermic Newborns}

Among the six newborns who were treated with systemic hypothermia, there was no change in the imaging pattern of injury between days 1 and 3 . Five subjects had normal imaging and one had focal brain injury. However, two of four hypothermic newborns imaged on day 10 demonstrated a major change in the dominant pattern of injury. Two newborns with normal imaging on day 3 developed a pattern of $\mathrm{BN}$-predominant

Table 1. Clinical features

\begin{tabular}{lcc}
\hline & \multicolumn{2}{c}{$N(\%)$ or median (IQR) } \\
\cline { 2 - 3 } Clinical characteristics & Normothermic $(n=18)$ & Hypothermic $(n=6)$ \\
\hline Male & $7(28 \%)$ & $4(67 \%)$ \\
Maternal infection & $14(78 \%)$ & $2(33 \%)$ \\
Documented fetal distress & $7(39 \%)$ & $6(100 \%)$ \\
Urgent cesarean section & $7(39 \%)$ & $3(50 \%)$ \\
Meconium & $40(38.4,40.7)$ & $2(33 \%)$ \\
Gestational age at birth & & $39(38,41.7)$ \\
(weeks) & $3,311.5(2,860,3,650)$ & $3,355(3,210,3,505)$ \\
Birth weight (g) & $6.98(6.87,7.1)$ & $6.98(6.83,7.08)$ \\
Cord pH & $15.8(19,11.3)$ & $9.2(12.6,8)$ \\
Base excess & $7.3(4.4,13)$ & $5.5(4.4,7.2)$ \\
Lactate (mmol/l) & $4(2,5)$ & $3(2,4)$ \\
Apgar, 5 min & $5(4,5)$ & $5(5,6)$ \\
Resuscitation score & $5(4,7)$ & $7(5,7)$ \\
Encephalopathy score & & $3(0,4)$ \\
(maximum days 1 and 3) & $3(0,5)$ & \\
Seizure score &
\end{tabular}

Summary of the clinical characteristics of newborns in the cohort. There were no significant differences between normothermic and hypothermic newborns using Kruskal-Wallis test for continuous variables or Fisher's exact test for categorical variables. $\mathrm{IQR}$, interquartile range. injury, whereas one newborn had persistent focal brain injury and one newborn had a normal MRI.

\section{Association of DTI and MRSI Values on Days 1 and 3}

There was a strong association of values from day 1 to 3 of life for nearly all parameters tested in the 19 newborns (14 normothermic and 5 hypothermic) evaluated with DTI and MRSI (Table 2). In gray matter, reduced mean diffusivity $\left(\mathrm{D}_{\mathrm{av}}\right)$ on day 1 was strongly associated with significantly reduced $\mathrm{D}_{\text {av }}$ on day $3(P<0.0001)$. Reduced ratios of $N$-acetylaspartate (NAA)/ choline $(P=0.04)$, as well as increased ratios of lactate/choline $(P=0.001)$ and lactate/NAA $(P=0.003)$ in gray matter on day 1 were also strongly predictive of ratios on day 3 . In white matter, reductions in $\mathrm{D}_{\text {av }}(P<0.0001)$, fractional anisotropy (FA) $(P=0.003)$, and NAA/choline $(P<0.0001)$ on day 1 were significantly associated with further reductions on day 3 .

Timing of MRI

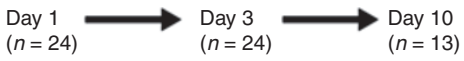

\begin{tabular}{|c|c|c|c|}
\hline $\mathrm{N}$ & $10-2$ & 61 & 0 \\
\hline $\mathrm{BN}$ & 3 & 3 & 3 \\
\hline$T$ & 1 & 3 & 2 \\
\hline F-MF & 3 & 5 & 3 \\
\hline WS & 1 & 1 & 1 \\
\hline
\end{tabular}

Hypothermic

\begin{tabular}{llll}
\hline $\mathrm{N}$ & 5 & 5 & 2 \\
\hline $\mathrm{BN}$ & 0 & 0 & 2 \\
\hline $\mathrm{F}-\mathrm{MF}$ & 1 & 1 & 1 \\
\hline
\end{tabular}

Figure 1. Evolution of the predominant pattern of injury on magnetic resonance imaging (MRI) in normothermic and hypothermic newborns on days 1, 3, and 10. BN, basal nuclei predominant; F-MF, focal-multifocal injury; N, normal; T, total; WS, watershed predominant. Arrows indicate the progression of the pattern of injury.

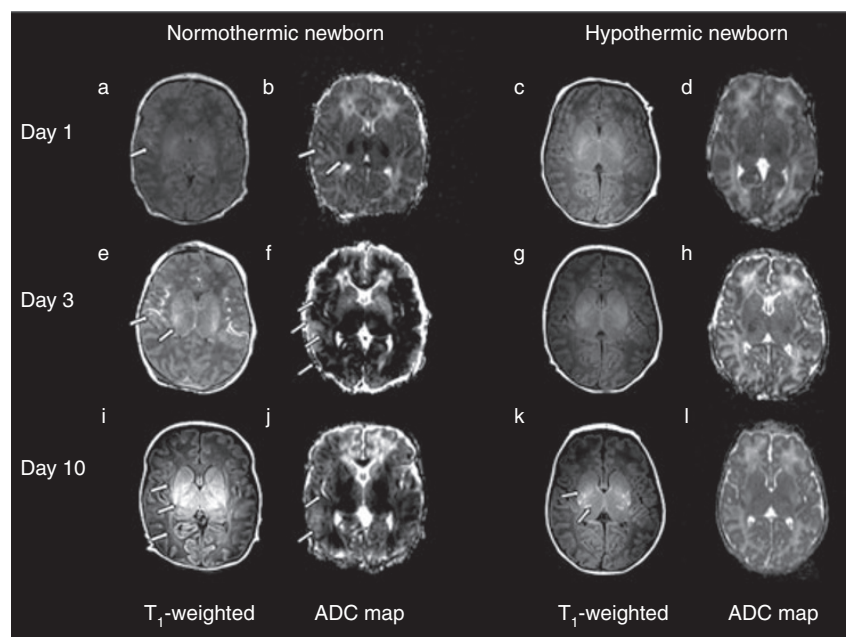

Figure 2. Progression of brain injury. Normorthermic newborn demonstrates $(\mathbf{a}, \mathbf{b})$ basal nuclei pattern on day $1,(\mathbf{e}, \mathbf{f})$ progression to total brain injury on day 3 , and (i,j) ongoing diffusion abnormalities on day 10 . Hypothermic newborn shows $(\mathbf{c}, \mathbf{g})$ normal $\mathrm{T}_{1}$ and $(\mathbf{d}, \mathbf{h})$ apparent diffusion coefficient (ADC) maps on days 1 and 3 . (k) Note the $T_{1}$ shortening in the posterior lentiform nuclei and ventrolateral thalami that develops on day 10 Area of signal abnormality indicated by white arrows. (I) Normal ADC map. 


\section{Association of DTI and MRSI Values on Days 1 and 10}

The association between DTI and MRSI values from day 1 to 10 of life was evaluated in 10 newborns (7 normothermic and 3 hypothermic) who had quantitative imaging repeated on day 10. In gray matter, reduced NAA/choline on day 1 was strongly associated with further reductions on day $10(P=0.001)$. In white matter, reduced $\mathrm{D}_{\text {av }}$ and FA (both $P<0.0001$ ), as well as ratios of NAA/choline $(P=0.04)$, on day 1 were predictive of further reductions on day 10 . Elevated ratios of lactate/NAA in white matter on day 1 were very strongly associated with persistently elevated lactate/NAA on day $10(P<0.0001)$.

\section{Association of Hypothermia With DTI and MRSI Values}

Hypothermia modified the relationship between DTI and MRSI values from day 1 to 3 of life (Table 3 ). In gray matter, hypothermia resulted in attenuation of the reduction of $\mathrm{D}_{\mathrm{av}}$ from day 1 to 3 (interaction $P=0.07$ ), as well as significantly reduced ratios of lactate/NAA (interaction $P=0.05$ ) and lactate/choline (interaction $P=0.04$ ) from day 1 to 3 . In white matter, hypothermia blunted the reduction of $\mathrm{D}_{\text {av }}$ from day 1 to 3 (interaction $P=0.009$ ) but had no apparent modulation of the metabolite ratios.

The associations of systemic hypothermia with DTI and MRSI values on day 3 were explored in a multivariate regression model if the interaction effect was not significant (Table 4). In gray matter, hypothermia was significantly associated with increased ratios of NAA/choline $(P=0.004)$ on day 3 . In white matter, hypothermia was significantly associated with increased FA $(P=0.001)$ and increased NAA/choline $(P=$ $0.007)$ on day 3 .

Because of the small number of newborns treated with hypothermia who were evaluated with quantitative imaging on day 10 , the effects of hypothermia on DTI and MRSI values on day 10 could not be explored in a multivariate model.

Table 2. Association of DTI and MRSI values on days 1 and 3 of life

\begin{tabular}{|c|c|c|c|c|c|c|}
\hline & \multicolumn{3}{|c|}{ Gray matter } & \multicolumn{3}{|c|}{ White matter } \\
\hline & Effect size & $P$ value & $95 \% \mathrm{Cl}$ & Effect size & $P$ value & $95 \% \mathrm{Cl}$ \\
\hline $\mathrm{D}_{\mathrm{av}}$ & 0.924 & $<0.0001$ & 0.503 to 1.346 & 0.583 & $<0.0001$ & 0.289 to 0.877 \\
\hline FA & & & & 0.346 & 0.003 & 0.12 to 0.573 \\
\hline NAA/choline & 0.554 & 0.04 & 0.0181 to 1.09 & 0.458 & $<0.0001$ & 0.223 to 0.693 \\
\hline Lactate/choline & 0.520 & 0.001 & 0.214 to 0.826 & 0.02 & 0.8 & -0.109 to 0.149 \\
\hline Lactate/NAA & 0.677 & 0.003 & 0.227 to 1.126 & 0.143 & 0.4 & -0.165 to 0.451 \\
\hline
\end{tabular}

Regression coefficient for quantitative diffusion and MRSI parameters on days 1 and 3. Linear regression for repeated measures was used to determine the association of quantitative diffusion and MRSI parameters from day 1 to 3, adjusting for age at the time of first scan and region of interest.

$\mathrm{Cl}$, confidence interval; $\mathrm{D}_{\mathrm{av}}$ mean diffusivity; DTI, diffusion tensor imaging; FA, fractional anisotropy; MRSI, magnetic resonance spectroscopic imaging; NAA, N-acetylaspartate.

Table 3. Effect modification of hypothermia on the association of DTI and MRSI values on days 1 and 3 of life

\begin{tabular}{|c|c|c|c|c|c|c|}
\hline & \multicolumn{3}{|c|}{ Gray matter } & \multicolumn{3}{|c|}{ White matter } \\
\hline & Interaction & $P$ value & $95 \% \mathrm{Cl}$ & Interaction & $P$ value & $95 \% \mathrm{Cl}$ \\
\hline $\mathrm{D}_{\mathrm{av}}$ & -0.491 & 0.07 & -1.018 to 0.0355 & -0.239 & 0.009 & -0.419 to -0.0592 \\
\hline NAA/choline & 0.064 & 0.8 & -0.445 to 0.573 & -0.182 & 0.4 & -0.635 to 0.271 \\
\hline Lactate/choline & -1.085 & 0.04 & -2.1 to -0.0692 & -0.025 & 0.9 & -0.486 to 0.436 \\
\hline
\end{tabular}

Summary table demonstrating the effect modification of hypothermia on the relationship between DTI and MRSI values on days 1 and 3 assessed using an interaction term.

$\mathrm{Cl}$, confidence interval; $\mathrm{D}_{\mathrm{av}^{\prime}}$ mean diffusivity; DTI, diffusion tensor imaging; FA, fractional anisotropy; MRSI, magnetic resonance spectroscopic imaging; NAA, N-acetylaspartate.

Table 4. Association of hypothermia with DTI and MRSI parameters on day 3 of life

\begin{tabular}{|c|c|c|c|c|c|c|}
\hline & \multicolumn{3}{|c|}{ Gray matter } & \multicolumn{3}{|c|}{ White matter } \\
\hline & Effect size & $P$ value & $95 \% \mathrm{Cl}$ & Effect size & $P$ value & $95 \% \mathrm{Cl}$ \\
\hline \multicolumn{7}{|l|}{$\mathrm{D}_{\mathrm{av}}$} \\
\hline FA & & & & 0.0511 & 0.001 & 0.021 to 0.081 \\
\hline NAA/choline & 0.139 & 0.004 & 0.045 to 0.233 & 0.108 & 0.007 & 0.03 to 0.186 \\
\hline Lactate/choline & & & & 0.0336 & 0.6 & -0.082 to 0.149 \\
\hline Lactate/NAA & & & & -0.0314 & 0.8 & -0.281 to 0.218 \\
\hline \multicolumn{7}{|c|}{$\begin{array}{l}\text { Summary table demonstrating the association of hypothermia with DTI and MRSI parameters obtained on day } 3 \text {. Linear regression for repeated measures was used to determine the } \\
\text { association of hypothermia with quantitative diffusion and MRSI parameters, adjusting for age at the time of first scan and region of interest. Only parameters with an interaction term } \\
\text { of } P>0.1 \text { were tested in these models. }\end{array}$} \\
\hline \multicolumn{7}{|c|}{$\mathrm{Cl}$, confidence interval; $\mathrm{D}_{\mathrm{av} \mathbf{v}^{\prime}}$ mean diffusivity; DTI, diffusion tensor imaging; FA, fractional anisotropy; MRSI, magnetic resonance spectroscopic imaging; $\mathrm{NAA}$, N-acetylaspartate. } \\
\hline
\end{tabular}




\section{DISCUSSION}

In this study, by comparing the relationship of pattern of injury and quantitative imaging parameters in term newborns with HIE uniformly scanned on days 1 and 3 of life, we found that brain injury is demonstrable on quantitative diffusion and spectroscopic imaging as it evolves in the initial hours after hypoxic-ischemic insult. Specifically, quantitative diffusion and MRSI parameters on day 1 of life were very strongly associated with those on day 3 in this prospective cohort of term newborns with HIE. There was also a strong relationship between DTI and MRSI parameters on days 1 and 10 of life, predominantly in the white matter. As qualitative imaging is commonly normal during the early evolution of hypoxic-ischemic injury, the application of quantitative MRI techniques to newborns with encephalopathy has important implications for the early diagnosis of hypoxic-ischemic injury (3,6-13). Moreover, the accurate detection of hypoxic-ischemic injury earlier in its evolution also has implications for the management and prognosis of newborns with $\operatorname{HIE}(3,4,8,12)$.

A study by Barkovich et al. prospectively imaged 10 term newborns with HIE using serial MRI, DTI, and proton MR spectroscopy over the first $2 \mathrm{wk}$ of life (3). The initial scan was obtained on day 1 in 4 of the 10 newborns, and the timing of the second scan varied from day 3 to 14 . The authors found that patterns of injury detected by standard anatomic imaging, DTI, and proton MR spectroscopy varied considerably during the first $2 \mathrm{wk}$ after injury. In accordance with the authors' observations, we were able to detect brain injury on quantitative imaging even when the qualitative MRI was normal on the third day of life. Given that patterns of injury can appear very different when performed at different time points, the uniformity of the timing of imaging in our cohort strengthens the significance of the association of findings between days 1 and 3 of life.

The predominant pattern of brain injury established on MRI in newborns with HIE is an important predictor of neurodevelopmental outcome (5). These data demonstrate that the primary pattern of injury established on day 1 was reproducible on day 3 in the majority of newborns in the cohort. Only normothermic newborns demonstrated a progression of the pattern of injury from day 1 to 3 . Ten of 13 newborns had the same pattern of injury on days 3 and 10. Whereas one normothermic newborn had minor focal white matter injury detected on day 10 after normal imaging, there was a delay in the development of BN-predominant injury on day 10 in two hypothermic newborns. The delayed development in the features of acute, profound asphyxia on the 10th day of life in these two hypothermic newborns suggests that the process of injury continues to evolve after cessation of hypothermia at $72 \mathrm{~h}$. In term newborns with HIE not treated with hypothermia, the predominant pattern of brain injury is robustly detected on the third day of life (15), but in those treated with hypothermia, the earliest time at which that predominant pattern is fully established still needs to be determined (16).

There were six newborns in our cohort treated with hypothermia as part of a randomized controlled trial (14) that was ongoing concurrent with the period of enrollment. More recently, systemic hypothermia has become standard of care in newborns with moderate to severe HIE. Data from randomized controlled trials have shown that $72 \mathrm{~h}$ of hypothermia initiated within $6 \mathrm{~h}$ of birth is associated with a significant reduction in neurodevelopmental disability at $18 \mathrm{mo}$ of age $(14,17,18)$. In our small subset of six hypothermic newborns, therapeutic hypothermia was associated with an attenuated reduction of $\mathrm{D}_{\mathrm{av}}$ in both the gray and white matter from day 1 to 3. Hypothermia was also associated with increased neuronal metabolism and white matter integrity, as well as reduced oxidative metabolism. Taken together, these data suggest that hypothermia altered the extent and progression of brain injury in our subset of newborns treated with therapeutic hypothermia. These findings are consistent with recently published studies that have shown that therapeutic hypothermia is associated with slower evolution of diffusion abnormalities on day 5 (19), as well as improved microstructure and metabolism in the deep gray nuclei on DTI and proton MR spectroscopy obtained on day 5 (13). Of note, our data extend these observations to the initial days of life during the period of active treatment with hypothermia.

Several studies have shown a decreased burden of injury on MRI in newborns with HIE treated with hypothermia (18-23). Given that systemic hypothermia is associated with decreased or no injury on qualitative MRI, quantitative metrics may have increased utility in newborns treated with hypothermia. Our data support the use of DTI and MRSI parameters as bridging biomarkers of the treatment effects of hypothermia in newborns with HIE (24). Early quantitative imaging may have particular prognostic relevance to newborns with sentinel events because this subgroup remains at risk of poor neurodevelopmental outcomes despite therapeutic hypothermia (25). In addition, our data are highly applicable to newborns who are not treated with hypothermia due to delayed diagnosis or contraindication to therapy.

There were some limitations to our study. It was challenging to obtain a research-based MRI on the first day of life in critically ill newborns. Although the size of the cohort was small, by studying the newborns at relatively uniform time points, variance in the statistical models was reduced, which enabled us to examine the effect of modification of hypothermia on the relationship between DTI and MRSI values on days 1 and 3 $(26,27)$. Imaging critically ill babies requires specialized equipment for transport to the MR scanner, as well as trained personnel to provide ongoing clinical care during MR scanning. These requirements may make early imaging of newborns with HIE technically challenging, potentially limiting the widespread use of MRI on day 1 of life.

\section{Conclusions}

In conclusion, quantitative diffusion imaging and MRSI findings on the first day of life were strongly associated with those on the third day in this prospective cohort of term newborns with HIE. In our small subset of newborns treated with systemic hypothermia, cooling appeared to attenuate both the 
severity and progression of brain injury. Further prospective studies are underway to determine the relationship of quantitative diffusion parameters and metabolite ratios measured on day 1 in newborns with HIE and neurodevelopmental outcome.

\section{METHODS}

\section{Study Population}

The University of British Columbia Clinical Research Ethics Board approved this study. Parental informed consent was obtained. The study population consisted of term newborns with HIE who were cared for between 2006 and 2009 at the Children's \& Women's Hospital of British Columbia, a provincial tertiary level neonatal referral center. All newborns $(n=24)$ were imaged with $\mathrm{T}_{1} / \mathrm{T}_{2}$-weighted MRI with DW-MRI on day 1 of life $(24 \pm 12 \mathrm{~h})$ as part of the research protocol. Nineteen newborns were imaged with DTI and MRSI on day 1 of life $(24 \pm 12 \mathrm{~h})$ as part of the research protocol. Newborns were imaged on day 3 of life $(72 \pm 12 \mathrm{~h})$ with $\mathrm{T}_{1} / \mathrm{T}_{2}$-weighted MRI with DW-MRI commensurate with standard of care at this center. DTI and MRSI were also performed on day 3 in 19 newborns as part of the research protocol. Follow-up imaging was obtained on day 10 of life in 13 newborns based on clinical indications.

Inclusion criteria comprised gestational age $\geq 36 \mathrm{wk}$ and clinically recognizable encephalopathy, and at least one of the following criteria: fetal distress at delivery, requirement for resuscitation at birth, Apgar score $\leq 5$ at $5 \mathrm{~min}$, and metabolic acidosis (umbilical artery $\mathrm{pH}<7.1$ or base deficit $>10$ ). Newborns with confirmed congenital malformations, genetic abnormalities, antenatal infections, or inborn errors of metabolism were excluded.

A subgroup of newborns in this study $(n=6)$ was treated with systemic hypothermia on the basis of enrollment in a randomized controlled trial concurrent to the period of study (14).

\section{MRI Studies}

MRI studies were performed without sedation using an MR-compatible neonatal incubator (Lammers Medical Technology, Luebeck, Germany) and a high-sensitivity specialized neonatal head coil (Advanced Imaging Research, Cleveland, $\mathrm{OH}$ ) to reduce patient motion, increase patient safety, and improve the signal intensity-to-noise ratio of the MR images. The examinations were carried out on a Siemens 1.5 Tesla Avanto using VB 13A software (Siemens, Berlin, Germany) and included the following sequences: 3 -dimensional axial and coronal volumetric $\mathrm{T}_{1}$-weighted images (repetition time (TR), $36 \mathrm{~ms}$; echo time (TE), $9.2 \mathrm{~ms}$; field of view (FOV), $200 \mathrm{~mm}$; slice thickness, $1 \mathrm{~mm}$; no gap), axial fast spin echo $\mathrm{T}_{2}$-weighted images (TR, 4,610 ms; TE, $107 \mathrm{~ms}$; FOV, $160 \mathrm{~mm}$; slice thickness, $4 \mathrm{~mm}$; gap, $0.2 \mathrm{~mm}$ ), and isotropic DW images $b=600$ and $700 \mathrm{~s} / \mathrm{mm}^{2}$, with apparent diffusion coefficient maps (TR, 3,300 ms; TE, $82 \mathrm{~ms}$; FOV $210 \mathrm{~mm}$; slice thickness, $4 \mathrm{~mm}$; gap, $0.5 \mathrm{~mm}$ ).

An experienced neuroradiologist (K.J.P.), blinded to the newborn's medical history, coded each MRI $\left(\mathrm{T}_{1} / \mathrm{T}_{2}\right.$-weighted and $\left.\mathrm{DW}-\mathrm{MRI}\right)$ according to previously published criteria (28). The extent of injury was scored $0-4$ in the basal ganglia/thalamic region and $0-5$ in the WS region.

Because the predominant pattern of brain injury on MRI has previously been found to be a strong predictor of neurodevelopmental outcome in neonatal HIE, the subjects were then classified according to their predominant pattern of injury: normal, WS, BN, total, and focal-multifocal injury (stroke or white matter injury) $(5,15,28)$. Of note, these focal and multifocal injuries were distinct in location from the abnormalities classified as the WS predominant (15).

\section{Diffusion Tensor Imaging}

DTI was acquired with a multirepetition, single-shot echo-planar sequence with 12 gradient directions (TR, 4,900 ms; TE, $104 \mathrm{~ms}$; FOV, $160 \mathrm{~mm}$; slice thickness, $3 \mathrm{~mm}$; no gap), three averages of two diffusion weightings of $b=600$ and $700 \mathrm{~s} / \mathrm{mm}^{2}$, and an image without diffusion weighting, resulting in an in-plane resolution of $1.3 \mathrm{~mm}$. The MR data were transferred to an offline Siemen's workstation for post-processing. From the DTI data, we computed $\mathrm{D}_{\mathrm{av}}$ (also called apparent diffusion coefficient) and FA bilaterally in the anterior and posterior WS white matter, corticospinal tracts in the centrum semiovale, corpus callosum, posterior limb of the internal capsule, optic radiations, caudate, putamen, ventrolateral thalami, calcarine region, and hippocampi. These regions of interest (ROIs) were measured by manual anatomic voxel placements by the same study investigator without knowledge of the locations of injury as determined by $\mathrm{T}_{1} / \mathrm{T}_{2}$ MRI and the coding of the predominant pattern of injury. Intrarater reliability with this method is high, as previously described (29).

\section{MRSI}

MRSI was acquired using multivoxel chemical shift imaging (TR, 1,500 ms; TE, $144 \mathrm{~ms}$; averaging, 4). The volume of interest $(50 \times 50 \times 10 \mathrm{~mm}$ thick) was placed at two levels in the brain: high centrum semiovale just above the body of the lateral ventricles (to exclude cerebrospinal fluid) and basal ganglia at the level of the foramen of Monro. All spectra were analyzed offline by a single observer with voxels $(6 \times 6 \times 10 \mathrm{~mm})$ centered bilaterally on eight predefined ROIs: anterior and posterior WS white matter, corticospinal tracts in the centrum semiovale, optic radiations, caudate, putamen, ventrolateral thalami, and the calcarine region (29). Only voxels with adequate signal intensity-tonoise ratio that were fully included in the volume of interest were considered for statistical analyses $(>90 \%)$. The mean ratios of NAA/choline, lactate/choline, and lactate/NAA were measured for each ROI. Intrarater reliability with this method is high, as previously described (29).

\section{Clinical Data Collection}

Medical records were reviewed systematically for details related to pregnancy, labor, delivery, and perinatal course, including a resuscitation, encephalopathy, and seizure score $(30,31)$.

\section{Data Analysis}

Statistical analysis was performed with Stata software version 9.2 (Stata, College Station, TX). Clinical characteristics of normothermic and hypothermic newborns were compared using Fisher's exact test and Kruskal-Wallis test for categorical and continuous data, respectively. To assess the relationship between DTI and MRSI values on days 1 and 3 of life, we used linear regression for repeated measures, adjusting for age at the time of first scan in hours and ROI. The effect modification (interaction) of hypothermia on the relationship between DTI and MRSI values on days 1 and 3 was assessed using an interaction term. For variables with an effect modification of $P>0.1$, we examined the association of hypothermia to DTI and MRSI values on day 3 using a multivariate regression model with hypothermia as a predictor variable, adjusting for age at the time of first scan in hours and ROI. The relationship between DTI and MRSI values on days 1 and 10 of life was assessed using linear regression for repeated measures, adjusting for age at the time of first scan in hours and ROI. No mathematical correction was made for multiple comparisons, and all comparisons have been reported.

\section{STATEMENT OF FINANCIAL SUPPORT}

This work was supported by the SickKids Foundation and Institute of Human Development, Child and Youth Health-Canadian Institutes of Health Research National Grants Program (XG 07034). S.P.M. is supported by the Bloorview Children's Hospital Chair in Paediatric Neuroscience, with previous support from a Canada Research Chair (tier 2) and Michael Smith Foundation for Health Research Scholar award. D.G. is supported by the University of British Columbia Clinician Investigator Program.

Disclosure: The authors declared no conflict of interest.

\section{REFERENCES}

1. Volpe JJ. Hypoxic-ischemic encephalopathy: clinical aspects. In: Neurology of the Newborn, 4th edn. Philadelphia: W.B. Saunders Company, 2008:400-80.

2. Phelan JP, Martin GI, Korst LM. Birth asphyxia and cerebral palsy. Clin Perinatol 2005;32:61-76, vi. 
3. Barkovich AJ, Miller SP, Bartha A, et al. MR imaging, MR spectroscopy, and diffusion tensor imaging of sequential studies in neonates with encephalopathy. AJNR Am J Neuroradiol 2006;27:533-47.

4. Boichot C, Walker PM, Durand C, et al. Term neonate prognoses after perinatal asphyxia: contributions of MR imaging, MR spectroscopy, relaxation times, and apparent diffusion coefficients. Radiology 2006;239:839-48.

5. Miller SP, Ramaswamy V, Michelson D, et al. Patterns of brain injury in term neonatal encephalopathy. J Pediatr 2005;146:453-60.

6. Thayyil S, Chandrasekaran M, Taylor A, et al. Cerebral magnetic resonance biomarkers in neonatal encephalopathy: a meta-analysis. Pediatrics 2010;125:e382-95.

7. Chau V, Poskitt KJ, Miller SP. Advanced neuroimaging techniques for the term newborn with encephalopathy. Pediatr Neurol 2009;40:181-8.

8. Barkovich AJ, Westmark KD, Bedi HS, Partridge JC, Ferriero DM, Vigneron DB. Proton spectroscopy and diffusion imaging on the first day of life after perinatal asphyxia: preliminary report. AJNR Am J Neuroradiol 2001;22:1786-94.

9. Ward P, Counsell S, Allsop J, et al. Reduced fractional anisotropy on diffusion tensor magnetic resonance imaging after hypoxic-ischemic encephalopathy. Pediatrics 2006;117:e619-30.

10. Hanrahan JD, Sargentoni J, Azzopardi D, et al. Cerebral metabolism within 18 hours of birth asphyxia: a proton magnetic resonance spectroscopy study. Pediatr Res 1996;39(4 Pt 1):584-90.

11. Penrice J, Cady EB, Lorek A, et al. Proton magnetic resonance spectroscopy of the brain in normal preterm and term infants, and early changes after perinatal hypoxia-ischemia. Pediatr Res 1996;40:6-14.

12. Hunt RW, Neil JJ, Coleman LT, Kean MJ, Inder TE. Apparent diffusion coefficient in the posterior limb of the internal capsule predicts outcome after perinatal asphyxia. Pediatrics 2004;114:999-1003.

13. Bonifacio SL, Saporta A, Glass HC, et al. Therapeutic hypothermia for neonatal encephalopathy results in improved microstructure and metabolism in the deep gray nuclei. AJNR Am J Neuroradiol 2012;33:2050-5.

14. Jacobs SE, Morley CJ, Inder TE, et al.; Infant Cooling Evaluation Collaboration. Whole-body hypothermia for term and near-term newborns with hypoxic-ischemic encephalopathy: a randomized controlled trial. Arch Pediatr Adolesc Med 2011;165:692-700.

15. Chau V, Poskitt KJ, Sargent MA, et al. Comparison of computer tomography and magnetic resonance imaging scans on the third day of life in term newborns with neonatal encephalopathy. Pediatrics 2009;123:319-26.

16. Wintermark P, Hansen A, Soul J, Labrecque M, Robertson RL, Warfield SK. Early versus late MRI in asphyxiated newborns treated with hypothermia. Arch Dis Child Fetal Neonatal Ed 2011;96:F36-44.

17. Jacobs S, Hunt R, Tarnow-Mordi W, Inder T, Davis P. Cooling for newborns with hypoxic ischaemic encephalopathy. Cochrane Database Syst Rev 2007:CD003311.
18. Edwards AD, Brocklehurst P, Gunn AJ, et al. Neurological outcomes at 18 months of age after moderate hypothermia for perinatal hypoxic ischaemic encephalopathy: synthesis and meta-analysis of trial data. BMJ 2010;340:c363.

19. Bednarek N, Mathur A, Inder T, Wilkinson J, Neil J, Shimony J. Impact of therapeutic hypothermia on MRI diffusion changes in neonatal encephalopathy. Neurology 2012;78:1420-7.

20. Bonifacio SL, Glass HC, Vanderpluym J, et al. Perinatal events and early magnetic resonance imaging in therapeutic hypothermia. J Pediatr 2011;158:360-5.

21. Rutherford M, Ramenghi LA, Edwards AD, et al. Assessment of brain tissue injury after moderate hypothermia in neonates with hypoxic-ischaemic encephalopathy: a nested substudy of a randomised controlled trial. Lancet Neurol 2010;9:39-45.

22. Inder TE, Hunt RW, Morley CJ, et al. Randomized trial of systemic hypothermia selectively protects the cortex on MRI in term hypoxic-ischemic encephalopathy. J Pediatr 2004;145:835-7.

23. Wintermark P, Hansen A, Gregas MC, et al. Brain perfusion in asphyxiated newborns treated with therapeutic hypothermia. AJNR Am J Neuroradiol 2011;32:2023-9.

24. Azzopardi DV, Strohm B, Edwards AD, et al.; TOBY Study Group. Moderate hypothermia to treat perinatal asphyxial encephalopathy. N Engl J Med 2009;361:1349-58.

25. Sarkar S, Donn SM, Bapuraj JR, Bhagat I, Dechert RE, Barks JD. The relationship between clinically identifiable intrapartum sentinel events and short-term outcome after therapeutic hypothermia. J Pediatr 2011;159: 726-30.

26. Maxwell SE. Sample size and multiple regression analysis. Psychol Methods 2000;5:434-58.

27. Wampold BE, Freund RD. Use of multiple regression in counseling psychology research: a flexible data-analytic strategy. J Counsel Psychol 1987;34:372-82.

28. Barkovich AJ, Hajnal BL, Vigneron D, et al. Prediction of neuromotor outcome in perinatal asphyxia: evaluation of MR scoring systems. AJNR Am J Neuroradiol 1998;19(1):161-6.

29. Chau V, Poskitt KJ, McFadden DE, et al. Effect of chorioamnionitis on brain development and injury in premature newborns. Ann Neurol 2009;66:155-64.

30. Miller SP, Weiss J, Barnwell A, et al. Seizure-associated brain injury in term newborns with perinatal asphyxia. Neurology 2002;58: $542-8$.

31. Miller SP, Latal B, Clark H, et al. Clinical signs predict 30-month neurodevelopmental outcome after neonatal encephalopathy. Am J Obstet Gynecol 2004;190:93-9. 